Measurement of improvement Feedback from 2 cohorts (42 trainees) was consistently positive with all trainees gaining insight into topics which are not usually covered during training. Having a bespoke course helped speakers to discuss examples of paediatric issues and their management. Limiting the number of trainees helped to promote discussion as intended.

Effects of changes By 2014, all level 3 trainees in our deanery had completed this course. Trainees completed a SIP and presented it to their group on the final day of the course. Trainees were encouraged to discuss their projects and offered support with the SIPs.

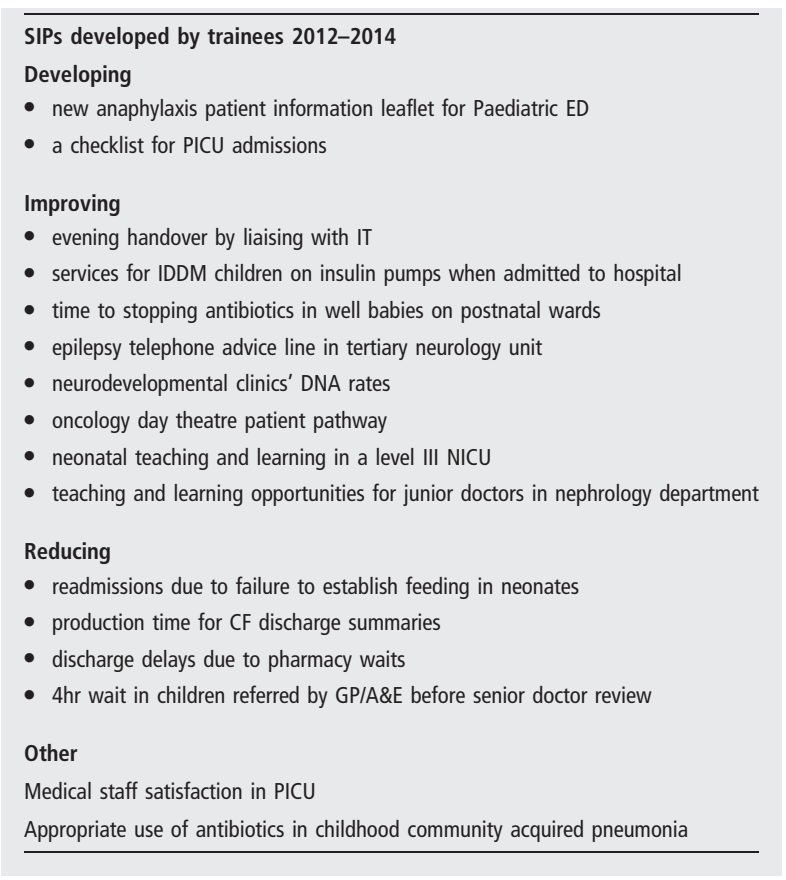

After review of feedback, we decided to run this course yearly for the next 5 years. The deanery has encouraged trainees to improve their understanding of clinical management by providing leave and funding. This has also helped individual hospital trusts by improving clinical care due to trainees SIPs.

Message for others We feel that early exposure to knowledge of clinical management will encourage trainees to help lead change in the future.

\section{G546(P) THE ASSESSMENT AND MANAGEMENT OF THE FEBRILE CHILD UNDER FIVE IN THE PRIMARY CARE SETTING}

A Andrews. Paediatrics, Royal Alexandra Children's Hospital, Brighton, UK

\subsection{6/archdischild-2015-308599.496}

Context This project was performed in a primary setting general practice surgery between October 2013 and January 2014. It involved analysis of data submitted by staff at the surgery including foundation doctors, registrar trainees, general practitioners and nurses.

Problem The aim of the project was to analyse the assessment and management of the febrile child under five years in a primary setting. This topic was chosen as infections was the main reason for children under five presenting to a general practice setting as well as being the leading cause of death. The National Institute for Health and Care Excellence (NICE) fever pathway was updated in May 2013 which provided a good framework for managing these cases and adherence to the fever pathway was assessed.

Assessment of problem and analysis of its causes According to the NICE fever pathway all children presenting with a fever should have their temperature, hear rate, respiratory rate and capillary refill time measured and recorded. Data was collected on the Egton Medical Information System (EMIS) retrospectively between May 2013 and August 2013 to determine whether this standard was adhered to. The results showed that only $10 \%$ of patients presenting with a fever has all parameters recorded.

Intervention In order to improve the documentation and assessment of the febrile child this data was presented to members of the practice in a team meeting highlighting the importance of measuring all the parameters in assessment of a child with a fever. A template was also designed to make this documentation more effective and presented to staff at the meeting. This template was added to EMIS and would be highlighted once the terms fever or temperature is documented on EMIS for any child under the age of 5 .

Study design The project was designed as an audit using the NICE fever pathway as the standard.

Strategy for change A teaching session was set up including all practice members. The data was presented as well as the recently improved NICE guidelines and traffic light system. Feedback from staff members on ways to improve data recording was encouraged through an informal discussion. The febrile child template was also presented to staff members showing them how it will be accessed and explaining how it should be used with reference to the NICE standards.

Measurement of improvement The impact of the intervention was determined by reassessing the data on EMIS after installing the febrile child template. Information entered on EMIS between November 2013 to January 2014 for each child presenting with a fever was analysed to determine if the assessment adhered to NICE standards.

Effects of changes The re-audit showed that following the teaching session on the assessment of the febrile child and the implementation of a template for recording the data there was a statistically significant improvement in assessing the febrile child with $62 \%$ having all parameters recorded in comparison to $10 \%$ on initial audit. This showed an improvement in management of these cases through accurate referrals to secondary care as well as appropriate use of antibiotics and safety net thus showing an overall improvement in clinical care.

Lessons learnt This project highlighted that simple interventions such as teaching sessions and the use of templates for recording data can improve clinical care of patients.

Message for others In busy clinical settings proformas and templates are helpful ways to standardise assessments and management of patients to ensure excellent standards of clinical care.

\section{G547(P) 'JUST CHECKING' - IMPLEMENTING A CHECKLIST}

1,2LC Budd, ${ }^{1,2} \mathrm{~L}$ Bignell, ${ }^{1,2} \mathrm{C}$ Bevan. ${ }^{1}$ Children's Emergency Department, Royal Alexandra Children's Hospital, Brighton, UK; ${ }^{2}$ Department of Paediatrics, Brighton and Sussex University Hospitals NHS Trust, Brighton, UK

\subsection{6/archdischild-2015-308599.497}

Context This quality improvement project as performed in a large District General Hospital, Children's Emergency Department (CED). It reviewed the 'Wheeze Pathway' used to treat children presenting with acute exacerbations of wheeze. These 
children initially receive "burst therapy" - 10 puffs of a Salbutamol inhaler via a spacer device every 20 min for $1 \mathrm{~h}$.

Problem A previous audit of the 'Wheeze Pathway' highlighted only $79 \%$ of children recieving burst therapy had their inhalers at the correct intervals and only $89 \%$ had appropriate observations. In a serious adverse event a child was transferred to the in-patient ward without a post burst therapy assessment, which necessitated eventual escalation to IV therapy and HDU admission. Within a busy CED it was difficult to easily establish which children were following the 'Wheeze Pathway' and at what time their inhalers, observations and assessments were due.

Assessment of problem and analysis of its causes Wheeze is a very common presentation to CED. Following the serious adverse event, nursing staff and doctors were consulted regarding the issues they felt contributed to the problems described.

Intervention Following a consultation process a 'Pathway Checklist' was developed (Figure 1). The checklist is a timeline of all treatments and assessments (medical and nursing) during the first $4 \mathrm{~h}$ of the 'Wheeze Pathway'. It was also decided children had to remain in the CED during this time to ensure a complete assessment with timely escalation of treatment if necessary and stability prior to transfer to a definitive care bed.

Strategy for change The 'Pathway Checklist' was developed with significant input from the nursing team to ensure strong support for the change. Upon implementation both the Pathway and Checklist were publicised to all staff and education provided at team meetings.

Measurement of improvement A snap shot review of the 'Wheeze Pathway' and 'Pathway Checklist' was undertaken over a 10-day period in 2014 and 39 consecutive sets of patient notes were reviewed.

\begin{tabular}{|c|c|c|}
\hline & Pre 'Pathway Checklist' & Post 'Pathway Checklist' \\
\hline Burst at correct intervals & $30 / 38(79 \%)$ & $37 / 39(95 \%)$ \\
\hline Observations after burst & $34 / 38(89 \%)$ & $39 / 39(100 \%)$ \\
\hline
\end{tabular}

The implementation achieved a near perfect number of children receiving burst therapy at the correct intervals and 100\% having complete observations as well as 37 (95\%) cases having timely medical reviews.

\begin{tabular}{lll} 
Abstract G547(P) & Table 2 & 'Pathway Checklist' Use \\
\hline Time interval & 'Pathway Checklist' completed & \\
\hline During burst therapy & 34 & $87 \%$ \\
Hour 2 & 29 & $74 \%$ \\
Hours 3 and 4 & 9 & $23 \%$ \\
\hline
\end{tabular}

Effects of changes As a consequence of its implementation there have been no further adverse incidents as children are now transferred to definitive care beds when stable and with clear plans. There is also now an unquantifiable improvement in how the department has a feeling of control and knowledge of all the children on the 'Wheeze Pathway', which has improved overall care of these patients. There is an allocated nurse to give all burst therapy and to provide education to families. There is also improved awareness amongst the nursing and medical teams

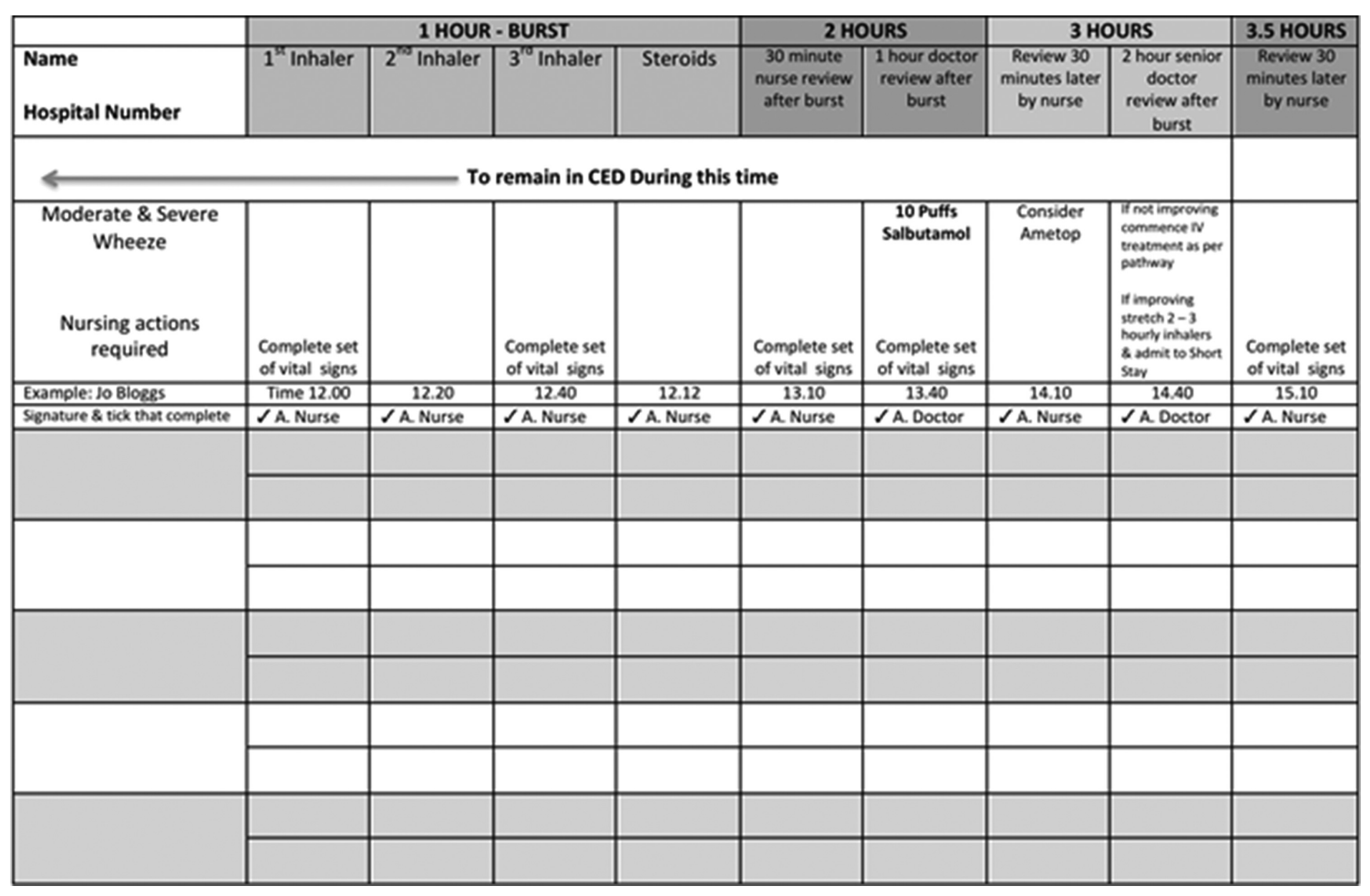

Abstract G547(P) Figure 1 Wheeze pathway checklist 